\title{
Potential of Thermally Expandable Polymers with Embedded Skeletons for Actuator Applications
}

\author{
Gih-Keong Lau ${ }^{a}$, Johannes F. L. Goosen ${ }^{b}$, Trinh Chu Duc ${ }^{c}$ and Fred van Keulen ${ }^{c}$ \\ ${ }^{a}$ School of Mechanical and Aerospace Engineering, Nanyang Technological University, \\ Nanyang Avenue, Singapore 639798 \\ ${ }^{b}$ Department of Precision and Microsystems Engineering, Faculty 3ME, Delft University of \\ Technology, Mekelweg 2, 2628 CD Delft, The Netherlands \\ ${ }^{c}$ DIMES, Delft University of Technology, Feldmannweg 17, 2628 CT Delft, The Netherlands
}

\begin{abstract}
This paper presents an overview of a new type of thermal micro-actuators using thermally expandable polymers with embedded skeletons. Embedding a stiff skeleton enhances the actuation capability of the thermally expandable polymer. Consequently, the skeleton-reinforced polymers feature a large maximum actuation stress (often above $100 \mathrm{MPa}$ ) and a moderate maximum strain (often above 1\%) besides a faster thermal response. In addition, the present composite design has room for performance improvement by tuning the volume fraction of the polymeric expander or selecting a proper expander material. Furthermore, the micro-actuators can be taylored for different motion characteristics, using various skeleton shapes. Finally, we discussed the possible applications using the present actuators.
\end{abstract}

Keywords: Thermally expandable polymer, thermal micro-actuators, material selection, design optimization, artificial muscles

\section{INTRODUCTION}

Thermally expandable polymers can deliver a high thermally-induced stress and a moderate thermally-induced strain. They can potentially deliver a large actuation energy. However, they are not directly available as actuation materials because they are both thermally and electrically insulating. However, thermally expandable polymers can be activated electro-thermally with an integrated heater. For example, a thin-film heater is commonly used to induce thermal expansion in thin layers of polymer. ${ }^{1,2}$ Typically, a thickness of a few tens of microns is used . However, a thin-film heater is not effective to heat up a thick polymer layer (with a thickness greater than 50 $\mu \mathrm{m})$. This results in a very limited thermal-mechanical actuation, which suffers from a slow thermal response and non-uniform heating. As a consequence, the thermal-mechanical actuation using polymers is not very useful at a larger scale (say $1 \mathrm{~mm}$ ).

Recently, we have shown that embedding a heat-conducting skeleton, in addition to a thin-film heater, in a $50-\mu \mathrm{m}$ thick polymer, can significantly improve heat transfer to the polymer. The skeleton has a large aspect ratio and thus a large contact area with the polymer, providing fast heat conduction. In addition, the embedded skeleton constrains the polymeric expansion laterally. It therefore enhances the expansion and stiffness of the polymer in the transverse direction $(\perp)$, i.e. the intended actuation direction. For example, a thermal microactuator design based on epoxy (SU-8) as a thermal expander and silicon as a skeleton, outperforms its constituent expansion materials in many aspects. As compared to the constituent epoxy, the composite design containing $50 \%$ volume fraction of epoxy improves thermal conductivity twwo and 370 times in the lateral and transverse directions, respectively. In addition, the composite design achieves 2.7 times higher actuation and 3 times higher stiffness than the bare epoxy while delivering as much thermal expansion as the bare epoxy. ${ }^{3}$

Further author information: (Send correspondence to G.-K. Lau or F. van Keulen)

G.-K. Lau: E-mail: gihkeong@gmail.com or mgklau@ntu.edu.sg

F. van Keulen: E-mail: a.vankeulen@tudelft.nl, Telephone: +31 (0)15 2786515

Electroactive Polymer Actuators and Devices (EAPAD) 2008, edited by Yoseph Bar-Cohen Proc. of SPIE Vol. 6927, 69271D, (2008) -0277-786X/08/\$18 $\cdot$ doi: 10.1117/12.776238 


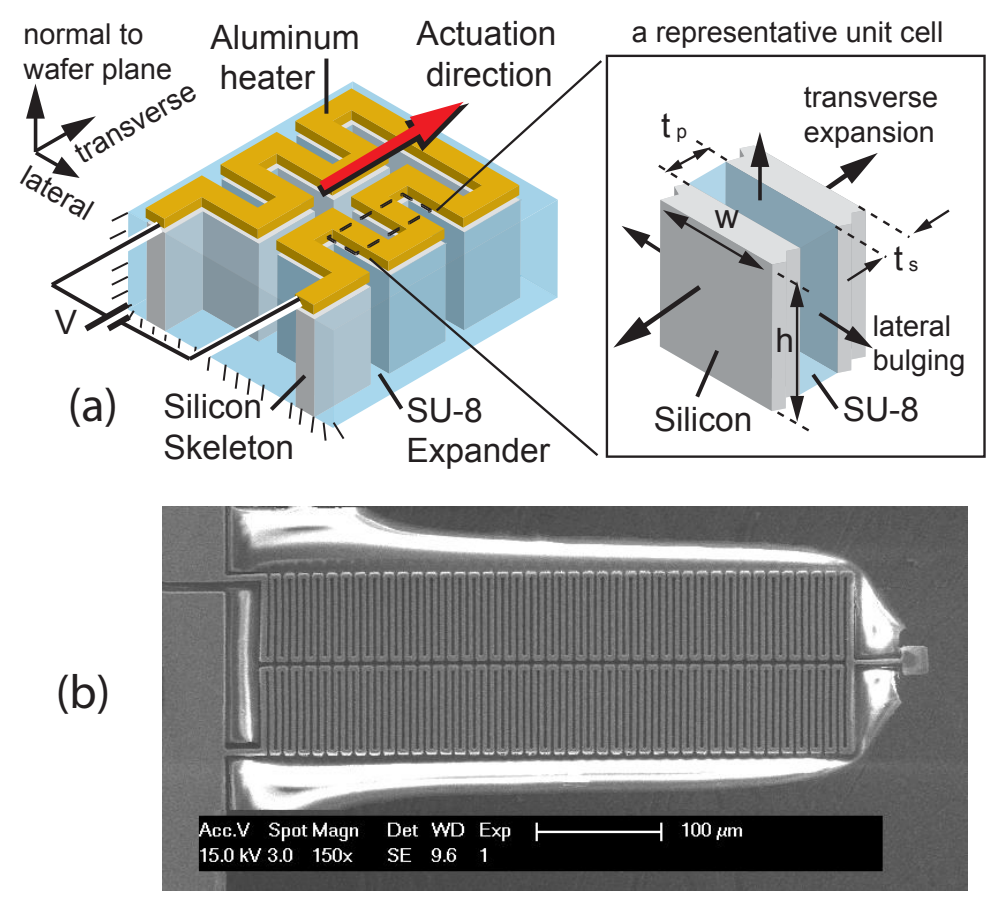

Figure 1. A polymeric actuator design with an embedded silicon skeleton: (a) a schematic drawing and a sandwiched model of laminae; (b) a scanning electron micrograph of a sample device (with a total length of $500 \mu \mathrm{m}$ and trench width of $3 \mu \mathrm{m})$.

The present paper will provide an overview on what has been and can be accomplished using the thermally expandable polymers with an embedded skeleton. We will first review the design principle that enhances the actuation capability. Then, we will discuss the room for design improvements by tuning the volume fraction of the polymer. Furthermore, we will explore various thermally expandable polymers and show their potential as the actuation materials. We will show some designs and their micro-fabricated devices based on the proposed actuation principle. Last, we will discuss the possible applications of the proposed actuator design.

\section{DESIGN PRINCIPLE}

A design example as shown in Fig. 1 is used to illustrate the effect of embedding a skeleton in a polymer block. The example design has a meander-shaped skeleton, which is resembles to a large extent an array of parallel plates. Provided the actuator width is sufficiently large as compared to the trench width, the design can be approximately modeled using a stack of polymer and skeleton layers. A representative unit of the stack is a polymeric layer perfectly bonded between two rigid plates. For simplicity, the stack is assumed to have an infinite width but a finite thickness. This approximate model can provide an insight into the working principle and the effect of parameter changes of the polymer actuator with the embedded skeleton.

The rigid plates, representing the skeleton, impose a lateral restraint on the deformation of the polymer layer and thus reinforces the latter. For a polymer layer, which is perfectly bonded between rigid plates with an infinite width, the apparent transverse Young's modulus $\left(E_{\perp}^{p}\right)$ is

$$
E_{\perp}^{p}=\left(\frac{1-\nu}{1-\nu-2 \nu^{2}}\right) E_{0}^{p}
$$

where $E_{0}^{p}$ and $\nu$ is Young's modulus and Poisson's ratio for an unconstrained polymeric layer.

Similarly, the lateral restraint directs volumetric expansion of the polymer in the transverse direction, leading to a higher apparent coefficient of thermal expansion (CTE). The transverse CTE $\left(\alpha_{\perp}\right)$ for the perfectly bonded 
layer between rigid plates of an infinite width is

$$
\alpha_{\perp}^{p}=\left(\frac{1+\nu}{1-\nu}\right) \alpha_{0}^{p}
$$

where $\alpha_{0}^{p}$ is the linear CTE for the unconstrained polymeric layer.

Both the apparent Young's modulus and CTE of the bonded layer are significantly higher than those of an unconstrained layer. The enhancement depends on the Poisson's ratio. In the extreme case where Poisson'ratio is 0.5 for an incompressible material, the stiffness enhancement increases to infinity whereas the CTE enhancement is 3 times higher than that of the unconstrained layer.

If a more sophisticated thermo-mechanical model is used to account for a lateral bulging together with the lateral restraint, the enhancement factors show dependence on the aspect ratio $(r)$ between the bond width $(w)$ and the layer thickness $\left(t_{p}\right)$. The effect of lateral restraint increases with the aspect ratio, reaching a maximum at the infinite width, as already estimated using Eq. 1 and Eq. 2. For more details on the dependence of the apparent properties on the aspect ratio, the interested readers are referred to Ref. ${ }^{4}$

In a composite stack of polymer and skeleton layers, the polymer component is the main contributer to the overall thermal expansion and elastic flexibility. Therefore, the composite properties depends on the volume fraction $(\phi)$ of the polymer component. According to the law of mixture, the composite Young's modulus $\left(E^{c}\right)$ and the composite CTE $\left(\alpha^{c}\right)$ in the transverse direction can be estimated by

$$
\begin{aligned}
& \alpha^{c} \approx \phi \alpha_{\perp}^{p} \\
& E^{c} \approx E_{\perp}^{p} / \phi
\end{aligned}
$$

Using these equations, we can estimate the effective thermal stress and work density for the composite of an infinite width and a finite thickness.

Furthermore, the embedded skeleton with a high thermal conductivity $\left(k^{s}\right)$ improves heat transfer to the insulating polymers with a low thermal conductivity $\left(k^{p}\right)$. Thus, the apparent thermal conductivity of the stack is anisotropic, where the transverse thermal conductivity $\left(k_{\perp}^{c}\right)$ and the lateral $\left(k_{\|}^{c}\right)$ can be estimated by

$$
\begin{aligned}
1 / k_{\perp}^{c} & =\phi / k^{p}+(1-\phi) / k^{s} \\
k_{\|}^{c} & =\phi k^{p}+(1-\phi) k^{s}
\end{aligned}
$$

Clearly, the composite has a higher thermal conductivity than the polymer alone and consequently it can response faster.

A maximum thermo-mechanical coupling factor, $\eta_{\max }$, can be defined as the ratio between the maximum thermo-elastic energy density over the heat capacity stored in a thermal expansion material, such that

$$
\eta_{\max }=\frac{E \alpha^{2} \Delta T_{\max }}{2 \rho c_{p}}
$$

where $E$ and $\alpha$ denote the Young's modulus and CTE of an expansion material, respectively; $\rho$ and $c_{p}$ denote the density and specific heat, respectively, while $\Delta T_{\max }$ denotes the maximum temperature rise. For a composite, the effective thermo-physical properties are used for the calculation, for example, $E=E^{c}, \alpha=\alpha^{c}$ and so on. This coupling factor reflects the maximum quantity of mechanical work that can be extracted from the expansion material given the input heat energy to induce the maximum temperature rise.

\section{DESIGN OPTIMIZATION}

As a composite material, the present actuator design has room for performance improvement by tuning the volume fraction of its constituents. The actuator performance is simulated using the simplified models, as discussed above and validated in Ref. ${ }^{5}$ using a fully-coupled multiphysics simulation.

To illustrate, we first evaluate a baseline design (see Fig. 1) consisting of $50 \%$ SU-8 as the polymeric expander, $50 \%$ silicon as the skeleton, and a thin-film aluminium heater. ${ }^{3}$ Table 1 shows that the actuator design delivers 
more energy in the actuation direction than the constituent materials on their own. The thermo-mechanical coupling factor of the $50 \%-50 \%$ SU-8/Si composite design is $1.44 \%$ as compared to $0.62 \%$ of SU-8 alone. Such performance improvement is tremendous and encourages further adoption of thermally expandable polymers as actuation materials.

Polymeric components largely determine the level of actuation performance, whereas the skeleton components mainly determine the effective thermal diffusivity and stiffness reinforcement. However, a $100 \%$ polymeric actuator design without skeleton material does not necessarily deliver better actuation than the one containing less polymer. This is because the polymeric strain enhancement caused by the skeleton confinement decreases with the polymeric content.

Consider a design space of $480 \mu \mathrm{m}$ long $\left(L_{0}\right), 50 \mu \mathrm{m}$ high $(H)$ and $60 \mu \mathrm{m}$ wide $(W=2 w)$. Let us explore possible design variations that fit into the design space, using a $3 \mu \mathrm{m}$ thick and the meandering skeleton of varying length. The parameter study shows that the design for maximum energy density has an optimum volume fraction of 70-30\% SU-8-Si. Table 2 summarizes performance comparison among the optimum design, the baseline design, and the polymer alone. ${ }^{6}$

The optimum design containing 70-30\% SU-8-Si improves energy density by a factor of 2.6, which is higher than 2.1 of the baseline design containing $50-50 \%$ SU-8-Si. In addition, the optimum composite design exhibits enhanced apparent CTE by a factor of 1.1, in comparison to 0.8 for the baseline design. Thermal stress for the optimum design is 2.5 times higher than the thermal stress for SU-8 alone. This value is slightly lower than that obtained by the baseline design, which reaches 2.6 times. Despite an increase of the insulating polymer content, the optimum design reduces the thermal response time by roughly $20 \%$, i.e. $97.7 \mathrm{~ms}$ as compared to $120.9 \mathrm{~ms}$ of the baseline design. This can be attributed to a shortened heater length.

Table 1. Apparent properteis and actuation capabilities, which are calculated using a lamellar model, ${ }^{3}$ for some thermal expansion materials.

\begin{tabular}{lllllll}
\hline & Symbols & Units & $\mathrm{Al}$ & $\mathrm{Si}$ & $\mathrm{SU}-8$ & $\begin{array}{l}\mathrm{SU}-8 / \mathrm{Si} \\
50 / 50 \%\end{array}$ \\
\hline Young's Modulus & $E$ & $\mathrm{GPa}$ & 70 & 130 & 3.2 & 9.5 \\
Poisson's ratio & $\nu$ & {$[1]$} & 0.36 & 0.28 & 0.33 & - \\
Coeff. Thermal Exp. & $\alpha$ & $\mathrm{ppm} / \mathrm{K}$ & 23.1 & 2.6 & 150 & 149 \\
Density & $\rho$ & $\mathrm{kg} / \mathrm{m}^{3}$ & 2700 & 2327 & 1200 & 1764 \\
Specific heat & $c_{p}$ & $\mathrm{~J} / \mathrm{kg} / \mathrm{K}$ & 904 & 702 & 1000 & 851 \\
Thermal conductivity & $k$ & $\mathrm{~W} / \mathrm{m} / \mathrm{K}$ & 237 & 148 & 0.2 & $0.4 / 74.1 \|$ \\
Actuation stress & $E \alpha$ & $\mathrm{MPa} / \mathrm{K}$ & 1.59 & 0.35 & 0.48 & 1.30 \\
Energy density & $\frac{1}{2} E \alpha^{2}$ & $\mathrm{~J} / \mathrm{m}^{3} / \mathrm{K}^{2}$ & 18.41 & 0.48 & 36.32 & 95.04 \\
Maximum temperature & $T_{\max }$ & ${ }^{\circ} \mathrm{C}$ & 660 & 1414 & 230 & 230 \\
Max. TM coupling & $\eta_{\max }$ & {$[\%]$} & 0.486 & 0.037 & 0.615 & 1.44 \\
\hline
\end{tabular}

\section{VARIOUS THERMALLY EXPANDABLE POLYMERS}

Besides the above-mentioned epoxy (SU-8), various other engineering polymers are thermally expandable, exhibiting a wide range of Young's moduli and CTE. These polymers can potentially be used as expansion materials. Some of them are photo-patternable while others are not. Among a larger set of polymers, we restrict our study to a few, because these polymers are commonly used for microelectronics and engineering application. Our study includes polyimide, benzocyclobutene (BCB), polymethylmethacrylate (PMMA), polyamide (Nylon), polyvinyliden fluoride (PVDF), Teflon polytetraluoroethylene (PTFE), and Vecra liquid crystal polymer (LCP).

Table 3 shows a list of the thermally expandable polymers and their intrinsic thermo-elastic properties. ${ }^{4}$ When the polymers are configured into a layer perfectly bonded between two rigid plates, their apparent properties can 
Table 2. Design variations and their performance for the polymeric actuators with an meandering skeleton. ${ }^{6}$

\begin{tabular}{llllll}
\hline Specification & Symbols & Units & $\begin{array}{l}100 \% \\
\text { SU-8 }\end{array}$ & $\begin{array}{l}50-50 \% \\
\text { SU-8/Si }\end{array}$ & $\begin{array}{l}* 70-30 \% \\
\text { SU-8/Si }\end{array}$ \\
\hline Volume fraction & $\phi$ & 1 & 1 & 0.5 & 0.7 \\
Actuator length & $L_{0}$ & $\mu \mathrm{m}$ & 480 & 480 & 480 \\
Actuator width & $W=2 w$ & $\mu \mathrm{m}$ & 60 & 60 & 60 \\
Actuator height & $H$ & $\mu \mathrm{m}$ & 50 & 50 & 50 \\
Thickness of skeleton segment & $t_{s}$ & $\mu \mathrm{m}$ & - & 3 & 3 \\
Thickness of polymer filling & $t_{p}$ & $\mu \mathrm{m}$ & 480 & 3 & 7 \\
Heater length & $L$ & $\mathrm{~mm}$ & $(6.84)$ & 10.46 & 6.84 \\
Apparent CTE & $\alpha^{c}$ & $10^{-6} / \mathrm{K}$ & 150.7 & 123.9 & 158.4 \\
Apparent Young's modulus & $E^{c}$ & $\mathrm{GPa}$ & 3.2 & 9.80 & 7.1 \\
Apparent actuation stress & $\sigma^{c}$ & $\mathrm{MPa} / \mathrm{K}$ & 0.48 & 1.25 & 1.18 \\
Apparent actuation energy & $\sigma^{c} \alpha^{c} / 2$ & $\mathrm{~J} / \mathrm{m}^{3} / \mathrm{K}^{2}$ & 36.3 & 77.8 & 93.8 \\
Response time & $\tau_{1}$ & $\mathrm{~ms}$ & $(229.5)$ & 120.9 & 97.7 \\
\hline
\end{tabular}

be estimated using Eq. 1 and Eq. 2. With the apparent properties and and the maximum temperature $\left(T_{\max }\right)$, we can estimate the maximum strain $\left(\epsilon_{\max }\right)$ and the maximum stress $\left(\sigma_{\max }\right)$.

Fig. 2 shows that, when configured in a layer perfectly bonded between rigid plates, SU-8, Nylon and LCP deliver maximum thermal stresses above $270 \mathrm{MPa}$. This suggests that the thermally expandable polymers can deliver very high actuation stress as compared to other means of actuation. ${ }^{7,8}$ For the actuation strain, we see that SU-8, PDMS and Teflon achieve maximum strains above $5 \%$. Among the polymers under investigation, PTFE (Teflon) achieves an extraordinarily high maximum thermal strain as much as $8.6 \%$. PTFE does not have a high intrinsic CTE, however, it has a Poisson's ratio very close to 0.5 . Consequently, PTFE manifests a substantial strain enhancement according to Eq. 2.

If these polymers are adopted as the expansion materials in the present actuator design, the actuator performance can be tuned to suit the required range of actuation stress and strain.

Table 3. Material data and maximum strain and stress for different polymeric thermal expansion materials.

\begin{tabular}{llllllll}
\hline Polymer type & $E_{0}^{p}$ & $\alpha_{0}^{p}$ & $\nu$ & $\begin{array}{l}T_{\max } \\
{ }^{\circ} C\end{array}$ & $\begin{array}{l}\epsilon_{\max } \\
\%\end{array}$ & $\begin{array}{l}\sigma_{\max } \\
\mathrm{MPa}\end{array}$ & $\begin{array}{l}\frac{1}{2} \epsilon_{\max } \sigma_{\max } \\
\mathrm{MJ} / \mathrm{m}^{3}\end{array}$ \\
\hline SU-8 & 3.2 & 150 & 0.33 & 220 & 5.8 & 275.3 & 8.00 \\
PMMA & 2.4 & 77 & 0.35 & 300 & 4.4 & 169.4 & 3.73 \\
Polyimde & 2.5 & 20 & 0.34 & 200 & 0.7 & 27.3 & 0.10 \\
PDMS & 0.002 & 310 & 0.2 & 130 & 4.9 & 0.1 & $2.6 \times 10^{-3}$ \\
PVDF & 2 & 150 & 0.34 & 169 & 4.4 & 135.0 & 2.96 \\
Nylon & 3.3 & 90 & 0.41 & 211 & 4.0 & 306.9 & 6.14 \\
BCB & 2.9 & 42 & 0.34 & 350 & 2.8 & 123.7 & 1.71 \\
Teflon PTFE & 0.46 & 135 & 0.46 & 260 & 8.6 & 182.4 & 7.82 \\
Vecra LCP & 10.6 & 30 & 0.35 & 280 & 1.6 & 270.3 & 2.14 \\
\hline
\end{tabular}




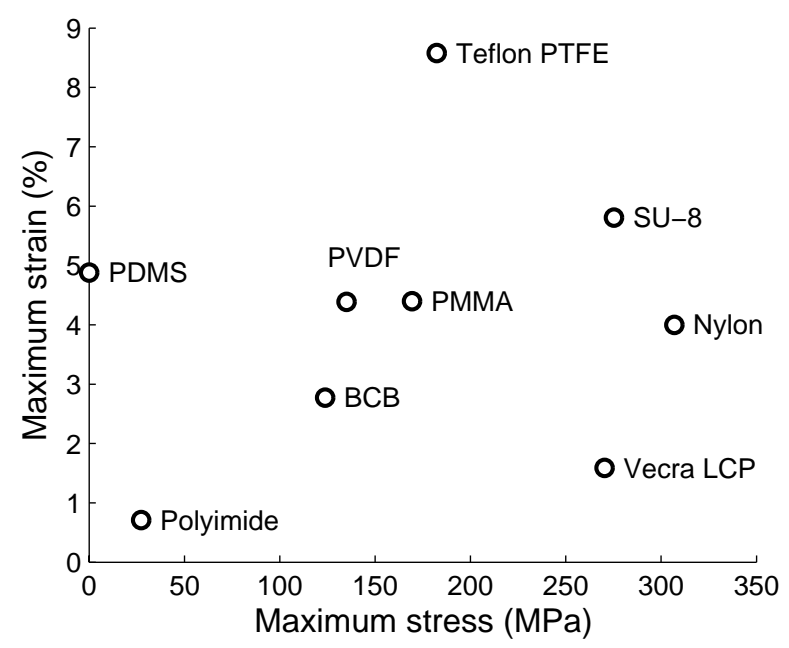

Figure 2. Maximum apparent strains and stresses for the polymers, which are configured as a layer perfectly bonded between two rigid plates.

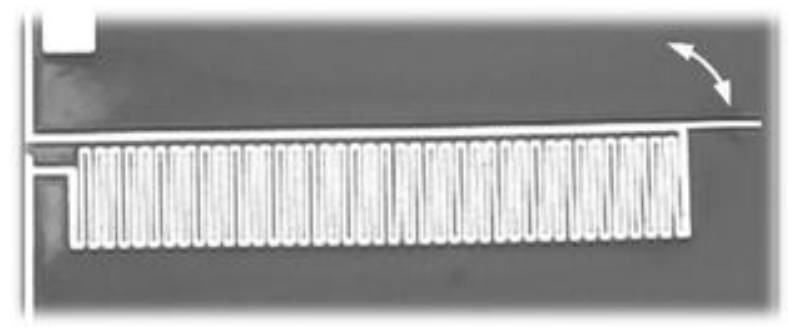

Figure 3. A polymeric actuator design with an embedded silicon skeleton of an asymmetric shape.

\section{FABRICATION}

So far, most polymers cannot be readily fabricated into high-aspect-ratio microstructure. However, silicon can be. For this reason, a high-aspect-ratio silicon microstructure, formed by bulk micromachining, is used to be a mold for micro-casting of the polymer.

Procedures of the fabrication are briefly described below, whereas the details can be found in Ref. ${ }^{9-11}$ For the heater, an aluminum film is first deposited by sputtering and then patterned by dry etching using a resist mask. The same resist mask is kept and used again for subsequent etching. Deep reactive ion etching (DRIE) of the silicon substrate forms cavities and trenches that define the shape of the silicon skeleton. Afterwards, a polymer in a liquid form, for example, the negative photo-resist of SU-8-2002, is cast and patterned to encase the silicon skeleton. Finally, the actuator micro-structure is released from the underlying substrate by backside etching using a nitride mask. 


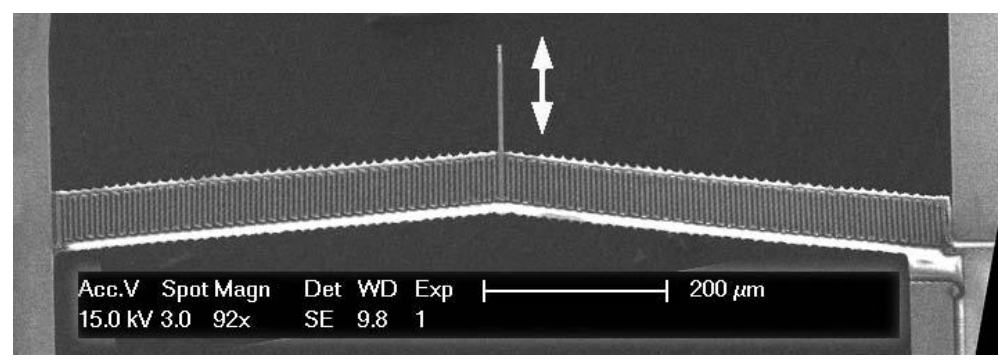

Figure 4. A polymeric actuator design with an embedded silicon skeleton of a V shape.

\section{OTHER SKELETON DESIGNS}

Various layout designs of skeletons have been developed for thermally expandable polymers. These include symmetric, asymmetric and V- shaped meandering skeletons. Polymer actuators with different skeleton layout could deliver varying characteristics of motion and force. The generated motion can be rectilinear, curvilinear, in-plane or out-of-plane.

Large displacements and blocked forces were clearly witnessed from the experiments and simulations for the various actuator designs. For example, a $530-\mu \mathrm{m}$ long polymeric bender with an embedded asymmetric skeleton ${ }^{12}$ (see Fig. 3) could easily generate a $25 \mu \mathrm{m}$ tip stroke, which is equivalent to a $30 \mathrm{mN}$ blocked force, at less than $2 \mathrm{~V}$, consuming less than $20 \mathrm{~mW}$ and operating below $200{ }^{\circ} \mathrm{C}$; a 1-mm long polymer actuator with embedded V-shape skeleton ${ }^{13}$ (see Fig. 4) could deliver a $25-\mu \mathrm{m}$ apex motion, equivalent to $135 \mathrm{mN}$ blocked apex force, at $2.5 \mathrm{~V}$, consuming $19 \mathrm{~mW}$ and operating at $177^{\circ} \mathrm{C}$.

It is noted that a thermally induced stress at a high temperature (above $220{ }^{\circ} \mathrm{C}$ ) may lead to fracture of the silicon skeleton. ${ }^{4}$ For example, the polymeric content in a V-shape actuator easily breaks one end of the embedded skeleton with a clamped-clamped boundary condition. Without doubts, the actuation capability using the integrated SU-8 expander and silicon skeleton are excellent.

\section{CONCLUSIONS AND POTENTIAL APPLICATIONS}

The presented study shows that the thermally expandable polymers with an embedded skeleton lead to powerful micro-actuators. These composite thermal actuators features a high actuation stress (often above $100 \mathrm{MPa}$ ) and a moderate strain (often above $1 \%$ ). They are especially suitable for applications where a large force generation and a compact size are required. Using the energetic expansion material, it is possible to pack many of these actuators in a high-density array. Looked upon as a composite material, the actuator designs provide a lot of room for performance improvement, by either geometry optimization or material selection.

Applications of the present actuator are not very obvious at the microscopic scale. So far, the actuator has been used to drive micro-grippers for particle micro-manipulation. ${ }^{10,11}$ However, a large force is not desired in micro-gripping because an excess in actuation has been observed to cause breakage to the jaw tips of the micro-grippers.

A potential application is forseen in micro-tensile testing where a large rectilinear force and an adequate displacement are required to pull or break a test specimen. Another potential application requires the actuator to dispense a fluid droplet, such as ink or medicine. In this application, the actuator needs to be configured as a membrane. The membrane actuator should generate out-of-plane motion at a certain driving pressure and speed. In addition, a bender using the proposed actuator design can be used to move a stiff optical fibre for alignment at a large force and an adequate displacement.

\section{ACKNOWLEDGMENTS}

The authors would like to thanks the Delft Center of Mechatronics and Microsystems for funding the present research project. In addition, the authors would like to thanks Professor P. M. Sarro for many discussions. 


\section{REFERENCES}

[1] Ataka, M., Omodaka, A., Takeshima, N., and Fujita, H., "Fabrication and operation of polyimide bimorph actuators for a ciliary motion system," J. Microelectromech. Syst. 2(4), 146-150 (1993).

[2] Nguyen, N.-T., Ho, S.-S., and Low, C. L.-N., "A polymeric microgripper with integrated thermal actuators," J. Micromech. Microeng. 14, $969-974$ (2004).

[3] Lau, G. K., Goosen, J. F. L., van Keulen, F., Chu Duc, T., and Sarro, P. M., "A powerful polymeric thermal micro-actuator with embedded silicon microstructures," Appl. Phys. Lett. 90, 214103 (2007).

[4] Lau, G. K., [Micro-electro-mechanical Actuators Using Confined Polymers], Delft University of Technology, The Netherlands (dec 2007). Ph.D. Dissertation, ISBN 978-90-9022531-9, Available Online at http://repository.tudelft.nl/file/773503/375528.

[5] Lau, G. K., Goosen, J. F. L., van Keulen, F., Chu Duc, T., and Sarro, P. M., "Polymeric thermal microactuator with embedded silicon skeleton: Part I design and analysis," submitted to J. Microelectromech. Sys. (2007).

[6] Lau, G. K., Goosen, J. F. L., and van Keulen, F., "Optimization of polymeric electro-thermal microactuator for various criteria," in [Proceedings of the 7th World Congress of Structural and Multidisciplinary Optimization], 902-911, Seoul, Korea (2007).

[7] Pelrine, R. E., Kornbluh, R. D., and Joseph, J. P., "Electrostriction of polymer dielectrics with compliant electrodes as a means of actuation," Sensors and Actuators A: Physical 64(1), 77-85 (1998).

[8] Madden, J. D. W., Vandesteeg, N. A., Anquetil, P. A., Madden, P. G. A., Takshi, A., Pytel, R. Z., Lafontaine, S. R., Wieringa, P. A., and Hunter, I. W., "Artificial muscle technology: physical principles and naval prospects," IEEE Journal of Oceanic Engineering 29(3), 706-728 (2004).

[9] Lau, G. K., Goosen, J. F. L., van Keulen, F., Chu Duc, T., and Sarro, P. M., "Electro-thermally activated polymeric stack for linear in-plane actuation," in [Proc. 5th IEEE Conference on Sensors], 538-541, Daegu, Korea (October 2006).

[10] Chu Duc, T., Lau, G. K., Wei, J., and Sarro, P. M., "Silicon-polymer laterally stacked bimorph microgripper," in [Technical Digest of the 17th MicroMechanics Europe (MME06)], 197-200, Southampton, UK (October 2006).

[11] Chu Duc, T., Lau, G. K., and Sarro, P. M., "Polymeric thermal micro-actuator with embedded silicon skeleton: Part II - fabrication and applications to microgrippers," submitted to J. Microelectromech. Sys. (2007).

[12] Lau, G. K., Chu Duc, T., Goosen, J. F. L., van Keulen, F., and Sarro, P. M., "In-plane thermal unimorph using confined polymers," J. Micromech. and Microeng. 17(7), S174-S183 (2007).

[13] Lau, G. K., Chu Duc, T., Goosen, J. F. L., van Keulen, F., and Sarro, P. M., "Power efficient V-shape electro-thermal actuator using constrained SU-8," in [Technical Digest of the 14th International Conference on Solid-State Sensors, Actuators and Microsystems (TRANSDUCERS'07)], 287-290, Lyon, France (June 2007). 